guns, clearly demonstrated the potentialities of some of the new boride emitters which operate under less stringent vacuum conditions. Looking forward for the next ten years, $\mathrm{Dr}$ Smith mentioned improvements in signal detection and the presentation of the final image, and gave some examples of the dynamic capabilities of the SEM. There then followed papers which described SEM at $8 \mathrm{~K}$ and at $1,600^{\circ} \mathrm{C}$, and Dr H. Yakowitz (National Bureau of Standards, Washington) gave details of a new high contrast cylindrical secondary electron collector which improves the signal quality from highly irregular surfaces.

The storage-display system described by Drs C. J. D. Catto and K. C. A. Smith (University of Cambridge) shows great promise, particularly where labile specimens susceptible to beam damage are being examined. Dr A. Boyde (University College, London) examined the problems associated with "real-time" three-dimensional examination of SEM images, a prerequisite for any meaningful analysis of morphological data.

The afternoon session, on information analysis, included papers on the $X$-ray microanalytical capabilities of SEM. It was made clear by Drs L. V. Sutfin (Harvard Medical School) and J. C. Russ (Edax Laboratories, N. Carolina) that high resolution X-ray microanalysis studies may be carried out on a wide range of materials, and further improvements may be expected in the sensitivity of energy dispersive $X$ ray detectors. The session also contained papers on crystallographic contrast, electron beam induced current in semiconductors and a review of the basic limitations of probe forming systems.

The second day started with a session on some of the techniques used in SEM. Dr P. Echlin (University of Cambridge) reviewed the present methods of thinfilm deposition onto non-conducting samples, and suggested a number of new coating techniques whereby more information could be obtained from specimens examined in the SEM. Dr $J$. Pawley (University of California at Berkeley) and Dr G. E. Pfefferkorn (University of Münster) considered the problem of surface charging, and there were several contributions on the nature of the electron beam interaction with the specimen. Dr E. K. Brandis (IBM, New York) described techniques for measuring height and depth, and the session finished with some of the specialized techniques required for the examination of radioactive material and semiconductors.

The final session was concerned with the physical limits of the scanning transmission microscope, and several speakers dealt with the theoretical capabilities of these instruments. Dr
J. M, Cowley (Arizona State University) described some of the design features of his $1 \mathrm{MeV}$ SEM, though the anticipated preliminary results from this instrument were not produced.

After the two day meeting there was a one day workshop sponsored by the National Institutes of Health, Washington, which concentrated on the problems of biological specimen preparation for SEM. The session was introduced by $\mathrm{Mr} \mathrm{E}$. Bynum (National Institute of General Medical Sciences), who spoke about the increased interest in scanning electron microscopy by the NIH and indicated that the agency is likely to increase its support for the medical and biological application of this type of instrumentation. After an excellent review by $\mathrm{Dr}$ A. Boyde (London), which dealt chiefly with the more conventional means of chemical fixation, there followed a series of contributions which demonstrated the advantages of cryobiological methods for specimen preparation. Problems of freezing and freeze drying were dealt with by Drs
A. P. MacKenzie (Cryobiology Research Institute, Madison) and $\mathbf{R}$. Thalmann (Washington University, St Louis), and Dr M. Nemanic (University of California, Berkeley) gave information relating to critical point drying and cryofracture. Much of the day was spent in detailing methods for particular specimens, and the papers on living tissue (Dr J. A. Nowell, University of California, Davis), plant material (Dr B. J. Panessa, New York University) and skin (Dr G. E. Pfefferkorn, Münster) were of particular interest.

Many manufacturers displayed their machines at the meeting, and it is noteworthy that most commercial instruments are now guaranteed better than $100 \AA$ in the secondary mode of operation. Some of the companies ran seminars in conjunction with the meeting, and the one day seminar by Kent Cambridge which preceded the meeting was particularly useful in demonstrating the wide range of analytical accessories which may be fitted to modern scanning microscopes.

\title{
Control of Serotonin Synthesis in Rat Brain
}

UNDERSTANDING of the biochemical mechanisms associated with the synthesis, storage and release of the catecholamine neurotransmitter substances has increased rapidly in recent years. Much less is known, however, about the biochemistry of the other monoamine transmitter, 5-hydroxytryptamine (5HT) (serotonin).

The way in which the rate of biosynthesis of the catecholamines noradrenaline and dopamine is controlled by nerve activity is now quite well defined. Such regulation seems to involve the biochemically familiar mechanism of end product feedback inhibition, in which the catecholamines control the first and rate-limiting step in their own biosynthesis, and the conversion of Ltyrosine to L-DOPA catalysed by the enzyme tyrosine hydroxylase. A report by Hamon et al. in next Wednesday's Nature New Biology (June 7) now provides evidence in favour of a similar mechanism to control the rate of biosynthesis of 5-HT in serotoninergic nerves in the rat brain.

Such a mechanism had been suggested previously by workers from this group on the basis of results obtained in the intact animal (Macon et al., $J$. Neurochem., 18, 323; 1971). In these experiments a decreased conversion of radioactively labelled tryptophan to 5HT in the brain was observed after treatment of rats with inhibitors of the enzyme monoamine oxidase to cause an increase in the steady state concentration of 5-HT. Because of the complexities involved in such experiments in the intact animal, however, it is reassuring to see that in Hamon et al.'s study similar effects could be observed in an isolated tissue preparation.

A reduced rate of conversion of labelled tryptophan to 5-HT was observed in slices of brain obtained from animals treated a few hours previously with pargyline or pheniprazine (monoamine oxidase inhibitors) when the tissue was incubated in saline medium containing the labelled amino-acid. Under such conditions, when the concentration of 5-HT in the tissue had increased to approximately 2.5 times the normal level, 5-HT formation was reduced by about 40 per cent. Evidence that this reduction in $5-\mathrm{HT}$ formation might be related to an increase in endogenous indoleamine concentration was confirmed by other experiments.

It thus seems that 5-HT may regulate its own biosynthesis by some form of end product inhibition. The precise nature of this inhibitory effect of 5-HT remains to be elucidated, but it does not seem to involve the rate of transport of the precursor amino-acid tryptophan into the serotoninergic neurones, because the presence of elevated concentrations of 5-HT did not reduce the specific activity of the tryptophan pool in Hamon et al.'s studies.

Hamon et al. conclude that the most probable mechanism is an inhibition of the first step in the biosynthetic pathway, the conversion of tryptophan to 5hydroxytryptophan, catalysed by the enzyme tryptophan hydroxylase. Such a conclusion must now await confirmation by studies with the isolated purified enzyme. 